\title{
A New Probabilistic Model of Language Competition, Endangerment and Extinction
}

\author{
Pritam Deka \\ Department of Computer Science and Engineering \\ Tezpur University \\ Assam, India
}

\author{
Smriti Kumar Sinha \\ Department of Computer Science and Engineering \\ Tezpur University \\ Assam, India
}

\begin{abstract}
Language endangerment is a growing concern in the world today. The speakers of the less dominant language in the society tends to get more inclined towards the more dominant language because of many reasons. Due to this, the speakers of the less dominant language decreases, eventually leading to the extinction of that languages.

In this paper, a probabilistic mathematical model has been proposed to check language shift for the intergenerational language transmission between parents and their offspring based on some factors that has been taken. A Markov chain model is also defined to show the change in language transmission for grandparent and grandchild.
\end{abstract}

\section{Keywords}

Language endangerment, Probability, Markov chain, Language shift, Absorbing state, Equilibrium vector, Transition matrix

\section{INTRODUCTION}

\subsection{What is a language}

According to Crystalinks[1], language is the complex system among human beings so as to exchange ideas and communicate with each other without having any difficulty.

Languagedevelopment [3] states that human beings "use language to express inner thoughts and emotions." We also use language so as to maintain the link with our culture and also to fulfil our daily needs.

\subsection{Language endangerment}

Ethnologue [2] states that "Language endangerment is a matter of degree." They have explained a scale of lower degree to higher degree where the less speaking languages are on the lower degree, these languages are endangered and some are on the verge of getting extinct if proper measures are not taken to help save these languages from getting wiped out from the earth.

A language may get endangered if the people speaking that language stops using that language and use the dominant language in the society in their homes with their children. As a result of which after some generations that language will lose its importance in the society and will eventually "die" out.

\section{ISSUES OF LANGUAGE ENDANGERMENT}

There are many issues regarding the endangerment of a language. A language can get endangered because of many reasons including political, social, and economic reasons. However, the endangerment of a language does not help the society much.

-Firstly, many important works of educational value also gets lost when the language gets endangered.

-A language is a way of communication among people of one culture. Part of the culture also dies out along with the language.

Many languages have already died out. Some are on the verge of extinction and yet others are endangered. This has led many socio-linguists to looks for ways to control the decreasing value of less dominant languages and to revive lost languages.

The best way to identify level of vitality of a language is still not defined properly. However, Ethnologue [2] has developed a scholarly consensus that can be applied globally and it may be possible in the near future to identify the level of vitality with the help of the consensus. Sociologists and linguistic anthropologists use various measures to identify trends in language use like number of speakers or use of language in certain domains. Also taken into consideration is the increase in bilingualism among people although a high level of bilingualism itself is not a sufficient condition for language shift or death.

\subsection{Evaluating Language Endangerment}

Ethnologue [2] reports data that are indicators of the two major dimensions of language use (users and functions); the number of users who identify with a particular language and the number and nature of the uses or functions for which the language is employed. A language may be endangered if fewer people use that language as their own heritage language and do not pass it to their next generations, rather shifts to a more dominant language.

Alternatively, it may also get endangered if it is being used in fewer social or economic activities.

Ethnologue [2] reports the following factors which may contribute to the assessment of language endangerment:

- The speaker population

- The ethnic population; the number of those who connect their ethnic identity with the language (whether or not they speak the language)

— The stability of and trends in that population size 
—Residency and migration patterns of speakers

- The use of second languages

- The use of the language by others as a second language

- Language attitudes within the community

- The age range of the speakers

- The domains of use of the language

-Official recognition of languages within the nation or region

-Means of transmission (whether children are learning the language at home or being taught the language in schools)

-Non-linguistic factors such as economic opportunity or the lack thereof

\section{RELATED WORK}

Many models have been suggested for modeling the language competition, death and survival. Here is a short survey of some models.

(1) Abrams and Strogatz Model:

Abrams and Strogatz [4] proposed a deterministic

mathematical model for language competition between two languages. For their model, they assumed a few things:

-Each individual speaks only one language and there is no bilingualism.

- The speakers of one language may switch to the other language based on a parameter "attractiveness".

- The attractiveness of a language increases with both the proportion of speakers of that language and its "status", a parameter that reflects the social or economic opportunities afforded to its speakers.

-A highly connected population, with no spatial or social structure.

- The population size remains constant.

They considered a system of two competing languages, $\mathrm{X}$ and $\mathrm{Y}$, in which the attractiveness of a language increases with both its number of speakers and its status. According to their model, if an individual converts from $\mathrm{Y}$ to $\mathrm{X}$ with a probability, per unit of time, $P_{y} x(x, s)$, where $\mathrm{x}$ is the fraction of the population speaking $\mathrm{X}$, and $0 \leqslant s \leqslant 1$ is a measure of $\mathrm{X}$ 's relative status, then a minimal model for language change can be defined as follows

$$
\frac{d x}{d t}=y P_{y} x(x, s)-x P_{x} y(x, s)
$$

where $y=1-x$ is the complementary fraction of the population speaking Y at time $t$. They assumed that by symmetry, interchanging languages should yield the same transition probability as a swap in the fraction of speakers and relative status; thus $P_{x} y(x, s)=P_{y} x(1-x, 1-s)$. They also assumed that no one will adopt a language that has no speakers $\left(P_{y} x(0, s)=0\right)$ or no status $\left(P_{y} x(x, 0)=0\right)$, and that $P_{y} x$ is smooth and monotonically increasing in both arguments. The assumptions suggest that equation (1) generically has three fixed points. Of these, only $x=0$ and $x=1$ are stable. The model therefore predicts that two languages cannot coexist stably and one will eventually drive the other to extinction.

The model is phenomenological rather being mechanistic, so it cannot define properly why language extinction occurs. It also does not incorporate bilingualism which is why the model has been criticized as bilingualism is a part of the society.

\section{(2) Minett and Wang Model:}

Minett and Wang[8] proposed an extension of the Abrams and Strogatz model in which bilingualism was introduced. After an initial proposal of the model in a working paper in 2005, they published the model which takes into consideration both the vertical and horizontal transmission of the languages [8]. This model focuses on language competence rather than use, and has seven free parameters, including prestige, volatility, four different peak transition rates, and a mortality rate [8]. However, they only present results regarding the case of neutral volatility $(a=1)$. In their review work of the Minett and Wang model, Castello et al. [9] summarized their results as follows

The first result shows that the language shift occurs only after the density of speakers of that language falls below a certain threshold value due to which the status of that language changes and so the shift takes place. However, by increasing the status of the endangered language or by adjusting the available educational resources in each of the languages, both the languages are maintained and can possibly coexist with non-negligible probability.

The second result takes into account a discrete society and then an agent based model is taken to study the society. They took into account fully connected networks and local world networks which was defined by $\mathrm{Li}$ and Chen[7] and then they have analyzed the frequency of convergence to each of the equilibrium points that was defined, based on the intervention threshold. They found similar results when they took into account only language coexistence, but they found that taking into account the factor of local world networks was more difficult to maintain.

\section{(3) Fernando et. al model:}

This model [5] of the mechanisms of language extinction and revitalization strategies to save endangered languages is a mechanistic model characterized by parameters that can be measured. Also, bilingualism has been incorporated into the model, so as to mainly focus on the functions of the child's family type and speaker frequency rather than describing the child's language type, monolingual or bilingual. One factor that has been defined in this model is language-frequencyindependent, i.e. the factor arises from explicit language teaching at schools. Also, they have distinguished the domain of language heard as public and private. The private domain is language-frequency-dependent. The model takes into account ways by which an endangered language can be saved from getting extinct and some intervention schemes which will help in the revitalization of the extinct languages. In agreement with the Wickström model [10], they have also observed that a strong tendency of individuals to learn both languages can result in stable bilingualism. The model also observes monolingualism-bilingualism coexistence for the correct choice of parameters, although the possible range of these parameters is narrow. The model does not observe any situation in which coexistence

depends on the initial values of the various languages; there is no threshold. However, the model observes a situation in which the choice of which language dominates in monolingualism depends on the initial ratio of high-status and low-status language use. Also, the model does not take into account the case of grandparent generation and how changes in language may take place in the long run. 


\section{THE PROPOSED MODEL}

The model that has been proposed is similar to that of the Fernando et al. model[5] in the way that it also takes into account the probability of the language spoken by the offsprings. It also takes into account the grandparent generation to check the shift in the language spoken by the different generations. It has been shown how changes can occur in the language spoken by grandparent and the grandchild and what may happen if changes continue to happen in the long run.

(1) Assumptions:

For defining the model, following assumptions has been made:

- Two languages $A$ and $B$ where $A$ is the major language spoken by majority of the population, $Y_{A}$ and $B$ is the minor language spoken by minority of the population, $Y_{B}$ and another portion of the popluation, $Y_{A B}$ can speak both the languages and we take it as $A B$.

- It is assumed that the grandparent generation(the paternal side) could speak either $A$ or $B$ but not both.

- It is assumed that $A$ speaking people can mate and have offsprings with $A$ speaking people only and the same applies to the $B$ speaking people but bilingual people can mate with any.

(2) Factors taken:

The factors used in the definition of the model are described below.

$\alpha_{h_{A}}$ : The contribution of language $A$ from home.

$\alpha_{c_{A}}$ : The contribution of language $A$ from community.

$\alpha_{h_{B}}$ : The contribution of language $B$ from home.

$\alpha_{c_{B}}$ : The contribution of language $B$ from community.

Also, $\alpha_{h_{A}}+\alpha_{h_{B}}=1$ and $\alpha_{c_{A}}+\alpha_{c_{B}}=1$

$\gamma=\frac{\omega_{B}}{\omega_{A}}$, is the language attitude ratio where,

$\omega_{B}$ : language attitude for language $B$, i.e., how much an individual will want to learn and speak language $B$ and $\omega_{A}$ : language attitude for language $A$. Also, $\omega_{A}, \omega_{B} \in[1,5]$ as it has been observed that stable values can be found for the probabilities within this range.

It is assumed that $\omega_{A} \neq 0$ as $A$ is the major language or the dominant language in the society.

$\omega_{B} \rightarrow 0, B$ may tend to 0 , which leads to the endangerment of the language $B$.

(3) The model:

The proposed model is a probabilistic model to find the language competition of two languages in a society.

The model can be defined in a mathematical way as follows. As the proportions of $A, B$ and $A B$ speaking people are assumed, the probability of speaking the language $A, P(A)$ and $B, P(B)$ can be found out using the model.

Now, since it has been assumed that only people who speak the same language can mate and bilingual people can mate with the others, so with that assumption the following 5 cases can be arrived at:

-A-A: both $A$ speaking people

-A-AB: $A$ speaking people mating with bilingual people

-B-B: both $B$ speaking people

-B-AB: $B$ speaking people mating with bilingual people

-AB-AB: both are bilingual

Depending on the above cases the following proportion of speakers are found respectively $Y_{A} Y_{A}, Y_{A} Y_{A B}, Y_{B} Y_{B}$, $Y_{B} Y_{A B}$ and $Y_{A B} Y_{A B}$.

As it is known that probability of any event happening is the ratio of the possible ways of that event happening to the total ways of the event happening, so $P(A)$ and $P(B)$ can be derived as follows:

$$
\begin{aligned}
& P(A)=\frac{Y_{A} Y_{A}+Y_{A} Y_{A B}+Y_{A B} Y_{A B}}{Y_{A} Y_{A}+Y_{A} Y_{A B}+Y_{A B} Y_{A B}+Y_{B} Y_{B}+Y_{B} Y_{A B}} \\
& P(B)=\frac{Y_{B} Y_{B}+Y_{B} Y_{A B}+Y_{A B} Y_{A B}}{Y_{A} Y_{A}+Y_{A} Y_{A B}+Y_{A B} Y_{A B}+Y_{B} Y_{B}+Y_{B} Y_{A B}}
\end{aligned}
$$

Based on these two probabilities, the probability of the language the offspring is going to speak for each case of the set of parents can be calculated as follows.

- Case 1: Both set of parents are $A$ speaking, i.e. A-A

Probability that offspring will speak $A$ can be found as

$P_{A_{A}}=\left(\alpha_{h_{A}}+\alpha_{c_{A}}\right) \cdot \frac{1}{\gamma} \cdot \frac{P(B)}{P(A)}, \begin{array}{r}0.6 \leq \alpha_{h_{A}}<1 \quad \text { for } \gamma \geq 1, \\ \alpha_{h_{A}}=1 \text { for } \gamma<1\end{array}$

As the major language spoken is $A$, we have taken the value of $\alpha_{h_{A}}$ to be in the range of 0.6 to 1 . And the probability that it will speak $B$ can be found out as

$$
P_{A_{B}}=\left\{\begin{array}{lr}
\alpha_{c_{B}} \cdot \gamma \cdot \frac{P(B)}{P(A)}, \quad \alpha_{h_{B}}=0 & \text { for } \gamma \leq 1 \\
\left(\alpha_{h_{B}}+\alpha_{c_{B}}\right) \cdot \frac{1}{\gamma} \cdot \frac{P(B)}{P(A)}, & 0.1<\alpha_{h_{B}} \leq 0.5 \\
\text { for } \gamma>1
\end{array}\right.
$$

Similarly, here as $B$ is the minor language and chances of speaking $B$ in this case of parent type is less, so we take the value of $\alpha_{h_{B}}$ to be in the range of 0.1 to 0.5 .

-Case 2: One set of parent speaks $A$ and other is bilingual, i.e. A-AB

Probability that offspring will speak $A$ can be found as

$$
P_{A_{A}}=\left(\alpha_{h_{A}}+\alpha_{c_{A}}\right) \cdot \gamma \cdot \frac{P(B)}{P(A}, \quad 0.6 \leq \alpha_{h_{A}} \leq 0.9
$$

As major language spoken in this case of parent type is $A$, so range of $\alpha_{h_{A}}$ is taken as given above. And the probability that it will speak $B$ can be found out as

$$
P_{A_{B}}=\left\{\begin{array}{rr}
\left(\alpha_{h_{B}}+\alpha_{c_{B}}\right) \cdot \gamma \cdot \frac{P(B)}{P(A)}, & 0.1 \leq \alpha_{h_{B}} \leq 0.4 \\
\left(\alpha_{h_{B}}+\alpha_{c_{B}}\right) \cdot \frac{1}{\gamma} \cdot \frac{P(B)}{P(A)}, & 0.1 \leq \alpha_{h_{B}} \leq 0.4 \\
\text { for } \gamma>1
\end{array}\right.
$$

Range of $\alpha_{h_{B}}$ will be as given above because it is the language spoken less in this case.

- Case 3: Both set of parents speak $B$, i.e. B-B

Probability that offspring will speak $A$ can be found as

$$
P_{B_{A}}=\left\{\begin{array}{lr}
\alpha_{c_{A}} \cdot \gamma \cdot \frac{P(B)}{P(A)}, \quad \alpha_{h_{A}}=0 & \text { for } \gamma \geq 1 \\
\left(\alpha_{h_{A}}+\alpha_{c_{A}}\right) \cdot \frac{1}{\gamma} \cdot \frac{P(B)}{P(A)}, & 0.1<\alpha_{h_{A}} \leq 0.5 \\
\text { for } \gamma<1
\end{array}\right.
$$

Since the major language spoken in this case is $B$, so range of $\alpha_{h_{A}}$ is less as given above and range of $\alpha_{h_{B}}$ will be more as shown below. And the probability that it will speak $B$ can be found out as

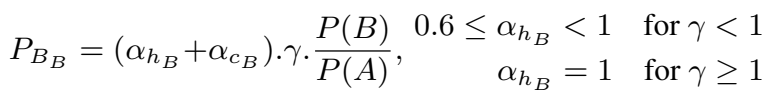


- Case 4: One set of parent speaks $B$ and other is bilingual, i.e. B-AB

Probability that offspring will speak $A$ can be found as

$$
P_{B_{A}}=\left\{\begin{array}{rr}
\left(\alpha_{h_{A}}+\alpha_{c_{A}}\right) \cdot \gamma \cdot \frac{P(B)}{P(A)}, & 0.1 \leq \alpha_{h_{A}} \leq 0.4 \\
\left(\alpha_{h_{A}}+\alpha_{c_{A}}\right) \cdot \frac{1}{\gamma} \cdot \frac{P(B)}{P(A)}, & 0.1 \leq \alpha_{h_{A}} \leq 0.4 \\
\text { for } \gamma<1
\end{array}\right.
$$

And the probability that it will speak $B$ can be found out as

$$
P_{B_{B}}=\left(\alpha_{h_{B}}+\alpha_{c_{B}}\right) \cdot \gamma \cdot \frac{P(B)}{P(A)}, \quad 0.6 \leq \alpha_{h_{B}} \leq 0.9
$$

Here also $B$ is the more spoken language among both so range of $\alpha_{h_{B}}$ is taken as such.

- Case 5: Both set of parents are bilingual, i.e. AB-AB

Probability that offspring will speak $A$ can be found as

$$
P_{A B_{A}}=\left(\alpha_{h_{A}}+\alpha_{c_{A}}\right) \cdot \frac{1}{\gamma} \cdot \frac{P(B)}{P(A)}, \quad 0.5 \leq \alpha_{h_{A}} \leq 0.9
$$

And the probability that it will speak $B$ can be found out as

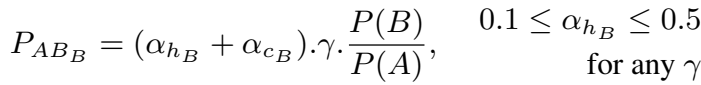

Since both are bilingual, so range is taken to be in a stable range which will define for both $A$ and $B$.

The model can be described as a method of calculating the probabilities of the language the offspring will speak for the various parent types that has been mentioned earlier. For all the cases that has been defined above, various probability values can be calculated which will help to understand better when language shift may occur or both languages will coexist.

To check the proposed model, a synthetic data set was taken and the model was simulated in Matlab. It has been found that conforming to the assumptions that has been taken and within the ranges defined for the factors, the stable point of coexistence for both the languages has been found to be for $\gamma=1$. This shows that when language attitude for both the languages are same then stable coexistence can be achieved. In real life situation also this is applicable as it can be seen that if both the languages are equally accepted by the people then both the languages can coexist. It has also been found that when the language attitude factor for language $B$ decreases the language curve tends to approach $\mathrm{X}$-axis which means it tends to "die". From the following figure where the case of $\boldsymbol{B}-\boldsymbol{B}$ speaking parents has been taken, it can be seen that after the stable point of coexistence for both the languages, curve for language $B$ decreases whereas for $A$ it increases as $\gamma$ decreases from 1 .

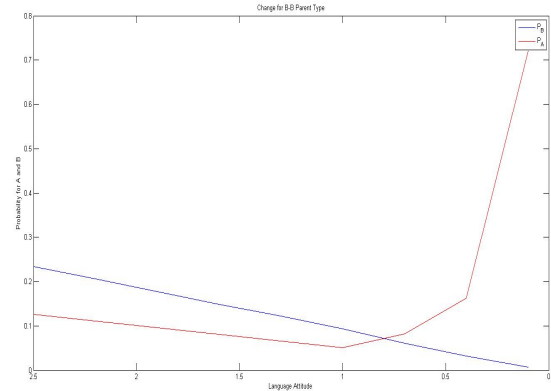

Fig. 1. Change in case of $B-B$ speaking parents

A basic working algorithm for the above model can be described as follows.

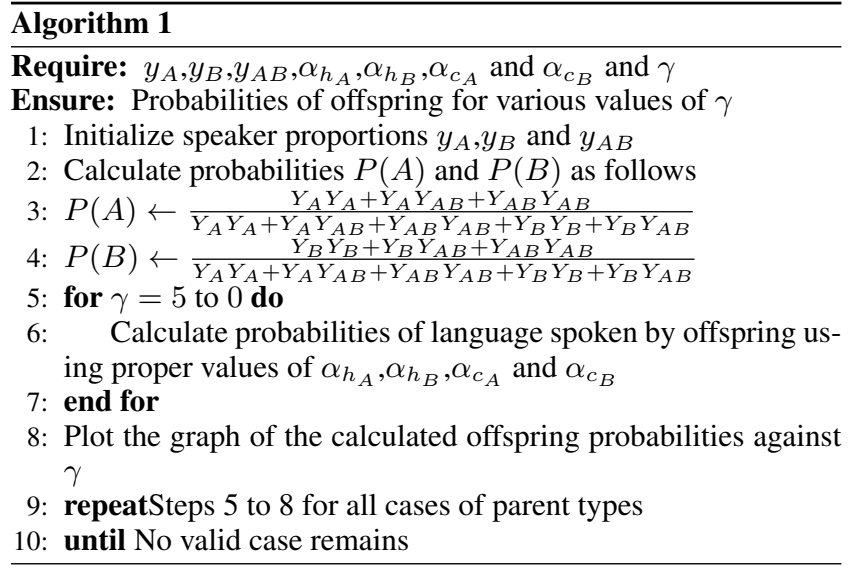

The intergenerational language transmission from the grandparent generation to the grandchild generation can be predicted using a markov chain which is a special type of stochastic process where the output of next state depends only on the present state and not on the previous states.

It can be shown that if grandparent is either $A$ speaking or $B$ speaking then depending on that the grandchild maybe $A$ speaking or $B$ speaking. The symbol $p_{i j}$ shows the transition from state $i$ to state $j$ in one generation. The transition diagram for both the languages can be shown as follows:

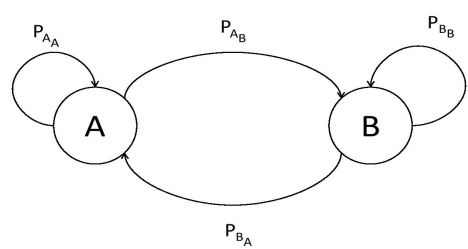

Fig. 2. Transition Diagram 
As defined by Kemeny and Snell[6], using this information a transition matrix can be defined for the generations. A transition matrix has the following properties:

(1) It is a square matrix

(2) All entries are between 0 and 1 as they are probabilistic values

(3) The sum of the entries in the rows gives the result of 1

Now we can define a transition matrix for the grandparent and grandchild generations. Such a matrix will also show two features of markov chain:

- the outcome of each set of experiment is a set of discrete states

- the outcomes depend only on the present state and not on the previous states

For the matrix that has been taken, a person is assumed to speak one of the two languages $A$ or $B$ and the offsprings are assumed to speak these two languages. This shows that discrete values are being taken. Also the change in language transmission can be shown by the following figures.

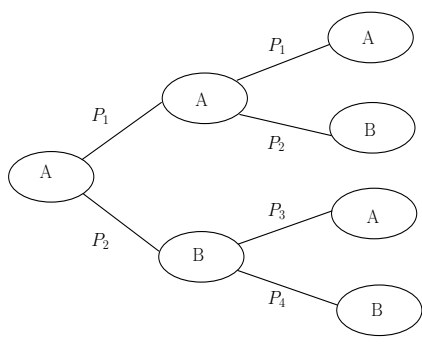

Fig. 3. Intergenerational Probability when Grandparent generation is $A$ speaking

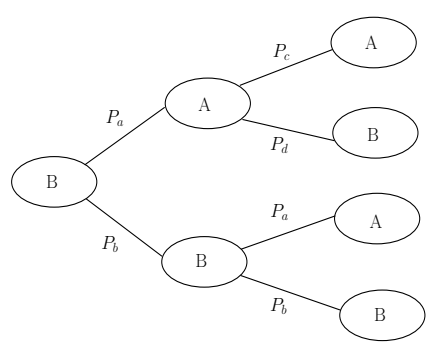

Fig. 4. Intergenerational Probability when Grandparent generation is $B$ speaking

For Fig 3 it is assumed that grandparent speaks $A$ and its offspring speaks either $A$ or $B$ with probability as shown in the diagram. As $p_{i j}$ has been used to denote the probability of changing from state $i$ to $j$ in one generation, this notation can be applied to find the probability of the grandchild speaking $A$. The probability can be written from the figure as follows:

$$
P_{\text {grandchild }_{A}}=P_{1} \cdot P_{1}+P_{2} \cdot P_{3}
$$

Similarly, the probability of grandchild speaking $B$ when grandparent speaks $A$ can be found as follows:

$$
P_{\text {grandchild }_{B}}=P_{1} \cdot P_{2}+P_{2} \cdot P_{4}
$$

The same way the probabilities for Fig 4 can also be found as well. The above sum of products of probabilities is nothing but the process of multiplying transition matrix $P$ by itself. So it can be said that $P^{2}$ gives the probability of state transition after two generations. An example matrix is taken to show the changes as described in the equations above. Since $A$ is the major language the probability $P_{1}$ from Fig. 1 can be taken as 0.9 and so $P_{2}$ will be 0.1 , let $P_{3}$ and $P_{4}$ be 0.6 and 0.4 respectively. So, the transition matrix $P$ will be :

$$
P=\left[\begin{array}{ll}
0.9 & 0.1 \\
0.6 & 0.4
\end{array}\right]
$$

From the equation (4), the probability of grandchild can be found by putting the values of $P_{1}, P_{2}, P_{3}$. After calculation it comes out to be 0.87 . Also after finding out $P^{2}$ the resultant matrix calculated is as follows:

$$
P^{2}=\left[\begin{array}{ll}
0.87 & 0.13 \\
0.78 & 0.22
\end{array}\right]
$$

It can be seen that the value of the matrix for $A$ is 0.87 which shows that our calculations are correct.

The change in proportion of speakers of language can also be shown in the same way for a table with the proportions of the speakers. The distribution of the proportions can be shown with the help of probability vectors which is a matrix of only one row having nonnegative values, with the sum of the entries equal to 1. If suppose the initial probability vector is $V_{0}$, then probability vector after one generation is $V_{0} . P$, after two generations is $V_{0} . P^{2}$ and so on. So it can be stated that for a Markov chain having initial probability vector

$$
V_{0}=\left[i_{1} i_{2} i_{3} \ldots i n\right]
$$

and transition matrix $P$ then probability vector after $n$ repetitions is

$$
V_{0} \cdot P^{n}
$$

Regardless of the initial probability vector, the system will approach a vector $V$ which will remain constant after that. As defined by Snell[6], this is the basic property of Markov chains: the limiting distribution is independent of the initial distribution. This happens due to the fact that some power of the transition matrix has all positive entries, which leads to the initial probability values getting mixed up. The vector $V$ is called fixed vector or equilibrium vector.

State $i$ of a Markov chain is absorbing state if $p_{i j}=1$. Now if a Markov chain enters such a state then it is not possible to leave. This definition can be extended to show the state of death in the case of language competition. If one language enters the absorbing state then it can be said that the language has "died". The absorbing Markov chain can be used to predict long term changes in the trend and if an absorbing state is reached then there is no returning back from that state. This can be applied to the concept of language competition as well where if the Markov chain somehow enters the absorbing state then it can be said that the language has become extinct or "died".

\section{CONCLUSION}

It can be seen from the resultant graph in Fig 1 , the plots for both the languages tend to decrease first as the $\gamma$ value decreases. But once it reaches the value 1 , then the language $B$ continues to decrease whereas $A$ increases. From this result it can be concluded that the assumptions in the model works correctly keeping in mind 
the societal aspects and its effects in the child's overall language attitude. Changes in the attitude of the language will determine the language shift. However, to be able to save the languages from getting extinct, the language $B$ has to be made popular among the speakers of $B$ so as to keep the language attitude of those speakers in a stable range. Such a case will lead to the coexistence of both the languages. There may be many ways to incorporate the popularity of $B$. Here, popularity may be taken as a factor and the language can be made popular by introducing it in the school level.

The proposed model works perfectly under the given assumptions, however, it may fail in cases where we want to generalize. Also, the proposed model does not take into account the bilingualism for the Markov chain which can prove to be a drawback as bilingualism is an integral part of society nowadays. The proposed model has scope for future improvements so that the dying language can be revitalized. Taking into account other possible factors, these factors can be incorporated into the model so as to find ways to revive extinct languages.

\section{REFERENCES}

[1] https://www.crystalinks.com/languages.html.

[2] https://www.ethnologue.com.

[3] http://www.languagedevelopment.tripod.com/id2.html.

[4] Daniel M Abrams and Steven H Strogatz. Linguistics: Modelling the dynamics of language death. Nature, 424(6951):900-900, 2003.

[5] Chrisantha Fernando, Riitta-Liisa Valijärvi, and Richard A Goldstein. A model of the mechanisms of language extinction and revitalization strategies to save endangered languages. Human biology, 82(1):47-75, 2010.

[6] John G Kemeny and J Laurie Snell. Markov chains.

[7] Xiang Li and Guanrong Chen. A local-world evolving network model. Physica A: Statistical Mechanics and its Applications, 328(1):274-286, 2003.

[8] James W Minett and William SY Wang. Modelling endangered languages: The effects of bilingualism and social structure. Lingua, 118(1):19-45, 2008.

[9] Marco Patriarca, Xavier Castelló, JR Uriarte, Víctor M Eguíluz, and Maxi San Miguel. Modeling two-language competition dynamics. Advances in Complex Systems, 15(03n04):1250048, 2012.

[10] Bengt-Arne Wickstrom. Can bilingualism be dynamically stable? a simple model of language choice. Rationality and Society, 17(1):81-115, 2005. 